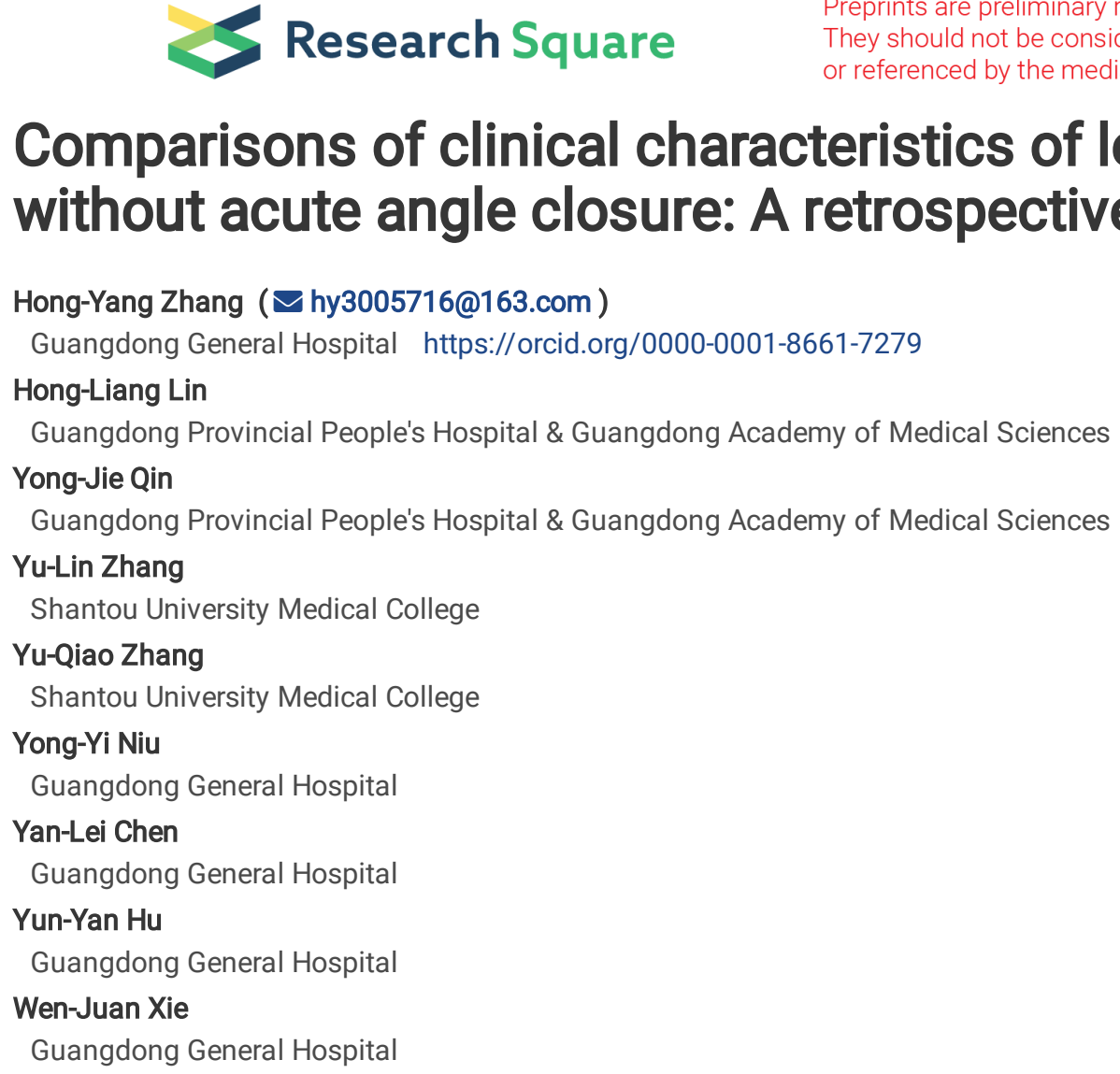

\title{
Comparisons of clinical characteristics of lens-subluxated eye with or without acute angle closure: A retrospective study
}

Keywords: Lens subluxation, zonular compromise, acute angle closure, anterior chamber depth

Posted Date: December 20th, 2019

DOI: https://doi.org/10.21203/rs.2.19423/v1

License: (c) (i) This work is licensed under a Creative Commons Attribution 4.0 International License. Read Full License 


\section{Abstract}

\section{Background}

To compare clinical characteristics of lens subluxation between eyes with or without acute angle closure (AAC).

\section{Methods}

This is a retrospective and case control study. Thirty-four cases with lens subluxation were recruited from 2015 to 2017 . Patients with acute angle closure were assigned to the AAC group ( $n=17$ eyes) and those without AAC were in the non-AAC group ( $n=17$ eyes). Quantitative anterior segment was evaluated by ultrasound biomicroscopy (UBM). Axial length (AL) was measured with IOL master. All patients underwent lens extraction surgery and were followed up for 6 months.

\section{Results}

The history of blunt trauma was accounted for 11 (64.7\%) cases in the AAC group and 14 (82.3\%) cases in the non-AAC group. Nine (52.9\%) patients in the AAC group had LPI or SPI treatment history, and high intraocular pressure was recurred. The UBM analysis showed that the average central ACD of affected eyes in the AAC group was $1.75 \mathrm{~mm}$, which was significantly shallower than the fellow eyes $(2.39 \mathrm{~mm}, P<$ 0.05 ) or both eyes in the non-AAC group (affected eye $3.24 \mathrm{~mm}$ vs fellow eye $3.81 \mathrm{~mm}$ ). Lens vault and AOD 500 also showed a remarkable difference between affected eye than fellow eye $(P<0.001)$ in the AAC group. The both eyes in the AAC group presented a shorter AL and shallower anterior chamber, comparing with those in the non-AAC group. Besides, the affected eyes in the AAC group presented significantly higher LV.

\section{Conclusions}

The crowded anterior chamber structure and shorter AL might be an anatomic basis for the eye with lens subluxation induced AAC. Quantitative evaluation of these ocular structures to identify zonular compromise, increased LV and shorter AL are valuable for the diagnosis of lens subluxation induced AAC.

\section{Background}

Many conditions can result in lens subluxation, including congenital, developmental, traumatic and iatrogenic zonulysis. The signs of lens subluxation include iridodonesis, phacodonesis, visibility of the lens equator, decentration of the lens, and vitreous prolapse in the anterior chamber. Lens subluxation can result in a decrease in the depth of the anterior chamber due to the forward movement of the dislocated lens, but not all the patients with lens subluxation have the obvious signs above mentioned. For the eyes with AAC attack caused by lens subluxation, it is difficult to determine the zonular stability before surgery and these signs may be neglected in the clinical setting[1]. A subluxated lens due to zonular weakness or partial loss may lead to pupillary block and sudden closure of the chamber angle[1]. It manifests with severe ocular pain, headache, elevated IOP, and shallow anterior chamber, which are similar to primary AAC. The eyes with lens subluxation induced AAC have no clinical signs of zonular weakness which appeared in the eyes with lens subluxation but without AAC[2]. Zonular dehiscence may be observed after full dilation of pupil by slit lamp examination, but it has a potential risk of incidental IOP elevation[3]. It is difficult to determine the zonular compromise in eyes with AAC attack before surgery. The role of the anteriorly positioned lens is an important factor in causing the acute angle closure[4, 5]. Except for anteriorly positioned lens, differentiating the differences of anatomic features is helpful to precisely diagnose the eyes with lens subluxation induced AAC and to avoid missed diagnosis. The present study was conducted to compare the clinical characteristics of lens subluxation between the eyes with or without AAC.

\section{Methods}

\section{Subjects}

We retrospectively reviewed 34 patients with unilateral lens subluxation recruited from the Department of Ophthalmology, Guangdong Provincial People's Hospital, from December 2015 to December 2017. All patients were divided into two group, according to with or without acute angle closure attack history. Seventeen eyes of 17 patients with lens subluxation and acute angle closure and their fellow eyes were in the AAC group. Seventeen eyes of 17 patients merely with lens subluxation and their fellow eyes were served as the non-AAC group. An informed consent was obtained from each patient before surgery. This study was approved by the institutional Human Research Ethics Committee of Guangdong Provincial People's Hospital and Guangdong Academy of Medical Sciences, Guangzhou, China and adhered to the tenets of the Declaration of Helsinki from the World Medical Association.

Inclusion criteria and exclusion criteria 
Acute angle closure was defined as[6, 7]: 1) Presence of at least two of the following symptoms: ocular or periocular pain; nausea and/or vomiting; and a history of intermittent blurring of vision with haloes; 2) Presenting IOP higher than $21 \mathrm{mmHg}$ in Goldmann applanation tonometry test; 3) Presence of at least three of following signs: conjunctival injection, corneal epithelial oedema, mid-dilated unreactive pupil, and shallow anterior chamber; 4) Presence of angle closure in the gonioscopy investigation. Patients with minimal to mild lens subluxation but without history or sign of previous acute angle closure were served as the non-AAC group.

Patients with following conditions were excluded: 1) Patients have been diagnosed with congenital diseases relating to lens subluxation, like the Marfan syndrome; 2) Patients have been definitely diagnosed as primary AAC; 3) Patients have clinically obvious or severe lens dislocation in the slit-lamp investigation; 4) Patients have undergone cataract surgery; 5) Patients are unable to cooperate with the ultrasound biomicroscopy (UBM) investigation. All patients received phacoemulsification operations and were followed up for 6 months.

\section{Examinations and treatments}

The degree of lens dislocation can be classified into 3 broad groups as described previously[8]: 1) Minimal to mild lens subluxation in which the lens edge uncovers $0 \%$ to $25 \%$ of the dilated pupil; 2 ) moderate lens subluxation in which the lens edge uncovers $25 \%$ to $50 \%$ of the dilated pupil; 3 ) severe lens subluxation in which the lens edge uncovers greater than $50 \%$ of the dilated pupil.

The medical histories were recorded for each patient. These histories included age, gender, family medical history, metabolic and genetic syndromes, ocular trauma, date of onset of symptoms, diagnosis, and treatments. All subjects underwent comprehensive ophthalmic examinations including assessment of Goldmann applanation tonometry, refraction tests, slit-lamp biomicroscopy, gonioscopy, dilated stereoscopic examination of the optic disc, and OCT (Spectralis OCT, Heidelberg Engineering, Germany). Uncorrected and corrected distance visual acuities (UDVA \& CDVA) were measured using the Snellen chart in decimal values. Spherical equivalent (SE) was calculated as a sphere plus half of the cylinder power. Gonioscopy, performed in a dark room (with and without indentation), was performed in all participants by a single examiner (ZHY), who was masked to the UBM findings, using a Goldmann 2-mirror lens (Ocular Instruments Inc., Bellevue, WA, USA). The angle in each quadrant was graded based on the observed anatomical structures, following the modified Shaffer grading system[9, 10] (grade 0, no structure or Schwalbe's line observed; grade 1, visible anterior nonpigmented TM; grade 2; visible posterior pigmented TM; grade 3, visible scleral spur; grade 4, visible ciliary body). Angle quadrants were considered as closed if they were grade 0 or 1 . Gonioscopic angle closure in an eye was defined as closure in 2 or more quadrants[11]. Quantitative parameters of anterior segment including the angle opening distance at 500 $\mu \mathrm{m}$ from the scleral spur (AOD500), anterior chamber depth (ACD), lens vault (LV), iris thickness at 750 $\mu m$ from the scleral spur (IT750), iris curvature (I-curve), and the status of the iris and zonules, were measured by the Ultrasound Biomicroscopy (UBM SW-3200L, Suoer Electrotec AG, China). In addition, axial length ( $A L)$ was measured by IOL Master (1322-734, Carl Zeiss Meditec AG, Germany). These procedures were performed by the same experienced ophthalmic technician (WJX). Qualitative UBM diagnoses were independently provided by two glaucoma ophthalmologists (HYZ and YJQ).

Preoperative IOL power calculations were performed using the AL and the keratometric readings measured by IOL Master. Phacoemulsification with capsular tension ring (CTR) and implantation of spherical IOLs with different diopters (Sensar AR40e, Abbott Medical Optics, CA, USA) were performed on those with 1 to 2 quadrants of zonular defect[12,13]. In the present study, there were 17 affected eyes in the AAC group and 15 eyes in the non-AAC group that received phacoemulsification with subsequent implantation of the intraocular lens (IOL) and the capsular tension ring (CTR). All the surgeries were done by a single surgeon (HYZ) uneventfully. No complications were found in a 6-month follow-up.

\section{Statistical analysis}

Descriptive statistics were calculated for demographic characteristics of the AAC group and the non-AAC group. To compare the means between two groups, parametric variables were analysed by using Student $t$-test. Analyses of nonparametric variables were done by the MannWhitney test. A $P$-value less than 0.05 was considered as statistically significant. All analyses were conducted by using SPSS version 20.0 (SPSS Inc., Chicago, Illinois, USA).

\section{Results}

\section{Demographic data}

Totally 34 eyes from 34 patients with lens subluxation were included in this study, the features of patients' history were summarized in Table 1. The age, gender, and history of blunt ocular trauma were not significantly different between the AAC group and the non-AAC groups ( $P \otimes 0.05)$. The duration since prior ocular trauma was significantly longer in patients with a history of AAC attack than in the non-AAC group ( $P=0.0166)$. When the patients were suffering from acute angle closure, ten (58.8\%) patients with lens subluxation induced AAC were misdiagnosed as primary AAC at their first attack. Nine (52.9\%) patients in the AAC group had LPI or SPI treatment history, and high intraocular pressure recurred. Before presented to our department, all patients with AAC had received treatments with anti-glaucoma medications for IOP

Page 3/9 
controlling. After removing the subluxated lens, the IOP was controlled without use of anti-glaucoma medications. As shown in Table 2, the elevated IOP in the AAC group was significantly decreased from pre-Op $39.46 \pm 16.71 \mathrm{mmHg}$ to post-0p $13.86 \pm 6.02 \mathrm{mmHg}(P<0.001)$ in a 6 month follow-up after surgery. The preoperative and postoperative IOP of affected eyes in the non-AAC group was in normal range (15.88 \pm 5.68 versus $14.82 \pm 5.70, P>0.05)$.

\section{Biometric features of lens subluxation in the AAC group and the non-AAC group.}

Quantitative analysis of UBM and gonioscopy of both eyes demonstrated a narrower anterior segment (ACD, AOD500 and gonioscopic grading) in the AAC group (Table 3). By analysing the 17 patients in the AAC group, we found that the central ACD of the affected eyes was significantly shallower than the fellow eyes $(1.75 \pm 0.62 \mu \mathrm{m}$ vs $2.39 \pm 0.65 \mu \mathrm{m}, P=0.006)$. As for peripheral anterior chamber, AOD 500 demonstrated a substantial decrease in the affected eyes when compared to the fellow eyes $(0.07 \pm 0.35 \mathrm{~mm} v \mathrm{~s} 0.25 \pm 0.14 \mathrm{~mm}, P \otimes 0.001)$. Moreover, LV in the affected eyes was remarkably higher than in the fellow eyes (1.59 $\pm 0.12 \mathrm{~mm}$ vs $0.57 \pm 0.75 \mathrm{~mm}, P \otimes 0.001)$. However, there was no significant difference in AL, I-curve or IT750 when comparing affected eyes and fellow eyes in the AAC group (P凶0.1). As shown in Figure, eye with lower modified Shaffer grade showed shallower ACD, smaller AOD500 and higher LV, compared to the fellow eye. However, there was no statistical difference of these parameters when comparing affected eyes and fellow eyes in the non-AAC group.

By comparing the AAC group and the non-AAC group, we found that both affected and fellow eyes in the AAC group showed shallower ACD, smaller AOD500, narrower anterior chamber angle, and shorter AL than those in the non-AAC group $(P<0.01$, Table 3$)$. The affected eyes of the AAC group and the non-AAC group showed significant differences in ACD, anterior chamber angle grading, AOD500, LV and AL (P < $0.01, T a b l e$ 3). It demonstrated that shallower ACD, smaller AOD500, higher LV and shorter AL are significantly associated with AAC attack.

\section{Discussion}

Zonular compromise can cause lens subluxation and was previously reported to occur from systemic anomalies such as Marfan syndrome, homocystinuria, and Ehlers-Danlos syndrome[14]. In the present study, these systemic diseases associated with obvious zonular dialysis were excluded. Moderate or severe lens subluxation could lead to a deepening of the anterior chamber with posterior dislocation of the crystalline lens, which was also excluded from this study. Only patients with minimal to mild lens subluxation was included in the AAC group and the zonular compromise between two groups was comparable. Lens subluxation may be acquired due to blunt external trauma or iatrogenic zonular damage. We found that $64.7 \%$ patients in the AAC group and $82.3 \%$ patients in the non-AAC group had a history of blunt ocular trauma, and eyes with AAC attack had a longer duration of prior ocular trauma histories than those in the non-AAC group (11.18 years vs. 1.47 years). It was reported that ocular blunt trauma can induce zonular damage in $42.9 \%$ patients[15]. The trauma, such as fist injury, door striking and ball hitting, was always ignored by the patients, though the history of ocular trauma was mainly dependent on the memory of the patient in the AAC group. So a more careful, trauma-focused history taking was suggested to help diagnose lens subluxation induced AAC[12]. Laser peripheral iridotomy (LPI) or surgical peripheral iridectomy (SPI) is effective to relieve the acute angle closure in primary AAC when pupil block occurs, but LPI or SPI is rarely effective in IOP reduction for patients with AAC secondary to lens subluxation[7, 16]. Lens subluxation induced angle crowding can lead to acute or chronic angle closure despite a patent peripheral iridectomy[17]. In this study, 9 eyes (52.9\%) in the AAC group had the LPI or SPI treatment history when they suffered previous acute attacks, but high pressure or AAC attack recurred. Our results showed that the anterior chamber became deeper after cataract surgery and the IOP was effectively controlled without anti-glaucoma medications for the patients in the AAC group.

The difference of anterior chamber depth between affected eye and fellow eye was detected in both groups by UBM. The asymmetric anterior chamber was obvious in the AAC groups. Our results showed that the average central ACD of affected eyes in the AAC group was 1.75 mm, which was significantly shallower than those in the fellow eyes $(2.39 \mathrm{~mm}, \mathrm{P}<0.05)$ or eyes in the non-AAC group (affected eye $3.24 \mathrm{~mm}$ vs fellow eye $3.81 \mathrm{~mm}$ ). Previously studies of eyes with AAC secondary to lens subluxation found that the ACD of the affected eyes was significantly shallower than that of their fellow eyes ( $1.34 \mathrm{~mm}$ vs. $2.27 \mathrm{~mm}$, and $1.29 \mathrm{~mm}$ vs. $2.12 \mathrm{~mm}$ ), consistent with the results in our present study $[1,12]$. The central ACD of affected eyes in the non-AAC group was slight shallow, but there is no statistical difference compared with the fellow eyes $(P>0.05)$.

Furthermore, analysis of AOD500, and LV also showed remarkable differences between affected eyes and fellow eyes in the AAC group. The measurement results of AOD500, LV were not statistically different as compared to their fellow eyes in the non-AAC group. Zonular compromise could lead to lens movement. Thicker and more anteriorly positioned crystalline lens showed LV increase, and higher LV may have a predominant role in AAC attack[18]. Lens thickness was not measured in our analysis. These results indicated that lens subluxation induced AAC generated greater central and peripheral ACD differences between affected and fellow eyes. However, no difference was found in the curvature, thickness and configuration of iris between affected eye and fellow eye in two groups. In addition, eyes of AAC group showed significantly shorter axial length when compared with those in the non-AAC group. Our study suggested a subluxated or forward tilted lens 
against the iris due to zonular compromise could lead to a shallow anterior chamber but crowded anterior chamber and shorter AL might be an anatomic basis for the eye with AAC caused by lens subluxation.

\section{Conclusions}

Lens subluxation can cause totally different clinical manifestations. Asymmetrical anterior chamber between both eyes is a representative and important feature in lens subluxation induced AAC. Quantitative evaluation of the ocular structures to identify zonular defects, lens vault increase, and shorter $\mathrm{AL}$ is valuable for the diagnosis of secondary AAC induced by lens subluxation. UBM is a valuable tool in assessing the degree of zonular compromise. The crowded anterior chamber structure and shorter AL might be an anatomic basis for the eye with AAC caused by lens subluxation compared with the eye without AAC attack.

\section{Abbreviations}

AAC, acute angle closure; LPI, laser peripheral iridotomy; SPI, surgical peripheral iridectomy; M/F, male/female; /: not applicable; Pre-Op, preoperative phacoemulsification; Post-Op, postoperative phacoemulsification. UDVA, uncorrected distance visual acuity; CDVA, corrected distance visual acuity; SE, spherical equivalent; IOP, intraocular pressure; D, diopters; Corn, cornea; S, sclera; AC, anterior chamber; I, iris; PC, posterior chamber; L, lens; LC, lens capsule; CB, ciliary body; T, temporal; N, nasal. ACD, anterior chamber depth; AOD500, angle opening distance at 500 $\mathrm{m}$ from scleral spur; LV, lens vault; I-curve, iris curvature; IT750, iris thickness at 750 $\mu \mathrm{m}$ from scleral spur; AL, axial length; LOCS III, Lens Opacities Classification System III; C/D ratio, Cup/Disk ratio; RNFL, retinal nerve fiber layer;

\section{Declarations}

\section{Acknowledgements}

Not applicable.

\section{Authors' contributions}

$\mathrm{HYZ}$, the corresponding author, filtering the cases, performed the standard surgical procedure and follow up the patients. HLL and YJQ were responsible for collection of data and wrote the major part of the study. YQZ and YLZ performed the statistical analysis and illustration. YYN, YLC, and YYH were responsible for interpretation of results. WJX was responsible for performing the standard examinations for all patients. All authors reviewed and approved the final manuscript.

\section{Funding}

The work was supported by the grants from the Natural Science Foundation of Guangdong Province, China (Ref No. 2018A030313833 to HYZ), and the National Natural Science Foundation of China (Ref No. 81600752 to YJQ).

\section{Availability of data and materials}

The datasets used and/or analyzed during the current study are available from the corresponding author on reasonable request.

\section{Availability of data and materials}

All data generated or analysed during this study are available from the corresponding author on reasonable request.

\section{Ethics approval and consent to participate}

All procedures performed in studies involving human participants were in accordance with the ethical standards of the Ethical committee of Guangdong Provincial People's Hospital and with the 1964 Helsinki declaration and its later amendments or comparable ethical standards. Informed written consent was obtained from all individual participants included in the study.

\section{Consent for publication}

Not applicable.

\section{Competing interests}

The authors declare that they have no competing interest. 


\section{References}

1. Kwon J, Sung KR: Factors Associated With Zonular Instability During Cataract Surgery in Eyes With Acute Angle Closure Attack. Am J Ophthalmol 2017, 183:118-124.

2. Guo S, Wagner R, Forbes B, Tannen B, Caputo A: Capsular tension ring in the management of occult lens zonular dehiscence in infantile glaucoma. J Pediatr Ophthalmol Strabismus 2010, 47 Online:e1-3.

3. Kim JM, Park KH, Han SY, Kim KS, Kim DM, Kim TW, Caprioli J: Changes in intraocular pressure after pharmacologic pupil dilation. $B M C$ Ophthalmol 2012, 12:53.

4. Moghimi S, Zandvakil N, Vahedian Z, Mohammadi M, Fakhraie G, Coleman AL, Lin SC: Acute angle closure: qualitative and quantitative evaluation of the anterior segment using anterior segment optical coherence tomography. Clin Exp Ophthalmol 2014, 42(7):615-622.

5. Suwan Y, Jiamsawad S, Supakontanasan W, Teekhasaenee C: Hidden mechanisms beyond the pupillary block in acute angle closure: ultrasound biomicroscopic study. Clin Exp Ophthalmol 2017, 45(4):366-370.

6. Ang LP, Aung T, Chew PT: Acute primary angle closure in an Asian population: long-term outcome of the fellow eye after prophylactic laser peripheral iridotomy. Ophthalmology 2000, 107(11):2092-2096.

7. Aung T, Ang LP, Chan SP, Chew PT: Acute primary angle-closure: long-term intraocular pressure outcome in Asian eyes. Am J Ophthalmol 2001, 131(1):7-12.

8. Hoffman RS, Snyder ME, Devgan U, Allen QB, Yeoh R, Braga-Mele R, Committee ACC, Challenging/Complicated Cataract Surgery S: Management of the subluxated crystalline lens. J Cataract Refract Surg 2013, 39(12):1904-1915.

9. Wang B, Sakata LM, Friedman DS, Chan YH, He M, Lavanya R, Wong TY, Aung T: Quantitative iris parameters and association with narrow angles. Ophthalmology 2010, 117(1):11-17.

10. Xu BY, Pardeshi AA, Burkemper B, Richter GM, Lin SC, McKean-Cowdin R, Varma R: Quantitative Evaluation of Gonioscopic and EyeCam Assessments of Angle Dimensions Using Anterior Segment Optical Coherence Tomography. Trans/ Vis Sci Technol 2018, 7(6):33.

11. Nongpiur ME, Aboobakar IF, Baskaran M, Narayanaswamy A, Sakata LM, Wu R, Atalay E, Friedman DS, Aung T: Association of Baseline Anterior Segment Parameters With the Development of Incident Gonioscopic Angle Closure. JAMA Ophthalmol 2017, 135(3):252-258.

12. Luo L, Li M, Zhong Y, Cheng B, Liu X: Evaluation of secondary glaucoma associated with subluxated lens misdiagnosed as acute primary angle-closure glaucoma. J Glaucoma 2013, 22(4):307-310.

13. Jacob S, Agarwal A, Agarwal A, Agarwal S, Patel N, Lal V: Efficacy of a capsular tension ring for phacoemulsification in eyes with zonular dialysis. J Cataract Refract Surg 2003, 29(2):315-321.

14. Dureau P: Pathophysiology of zonular diseases. Curr Opin Ophthalmol 2008, 19(1):27-30.

15. McWhae JA, Crichton AC, Rinke M: Ultrasound biomicroscopy for the assessment of zonules after ocular trauma. Ophthalmology 2003, 110(7):1340-1343.

16. Saw SM, Gazzard G, Friedman DS: Interventions for angleclosure glaucoma: an evidence-based update. Ophthalmology 2003, 110(10):1869-1878; quiz 1878-1869, 1930.

17. Epstein DL: Diagnosis and management of lens-induced glaucoma. Ophthalmology 1982, 89(3):227-230.

18. Zhang X, Liu Y, Wang W, Chen S, Li F, Huang W, Aung T, Wang N: Why does acute primary angle closure happen? Potential risk factors for acute primary angle closure. Surv Ophthalmol 2017, 62(5):635-647.

\section{Tables}


Table 1. Comparison of demographic and clinical data of the participants.

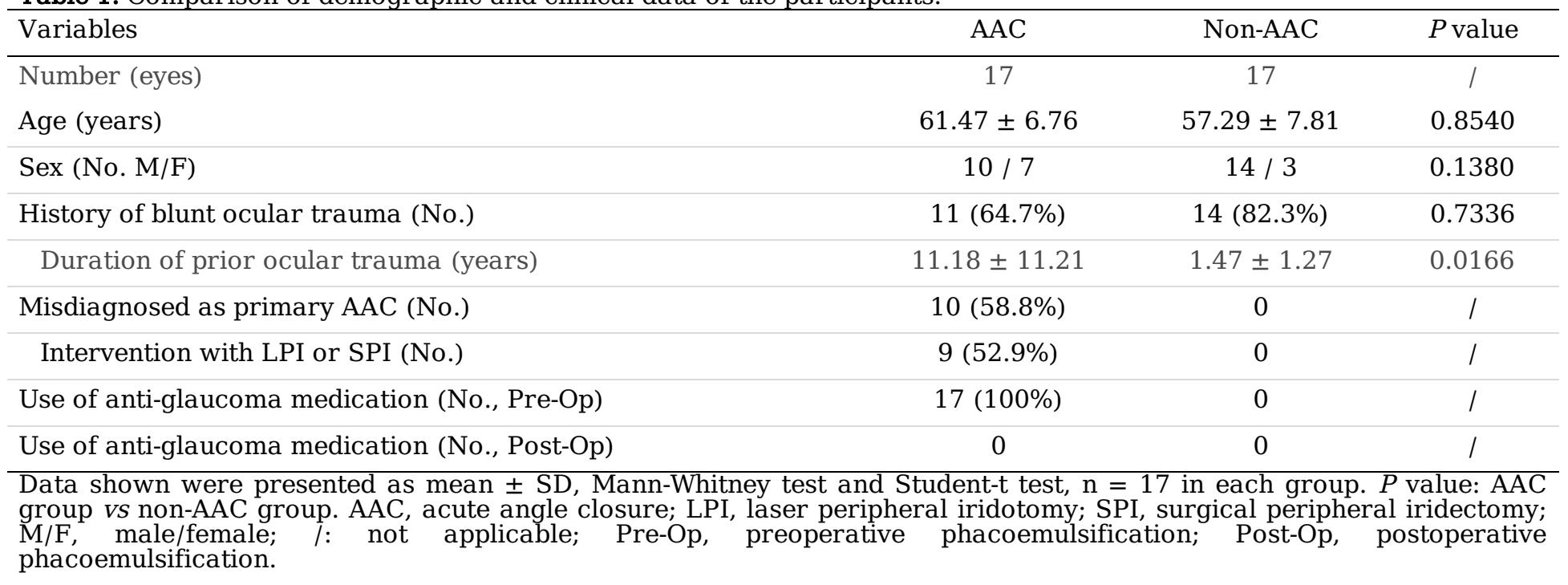

Table 2. Comparison of visual acuity and intraocular pressure in the affected eyes with lens subluxation.

\begin{tabular}{|c|c|c|c|c|c|c|}
\hline \multirow{2}{*}{ Parameters } & \multicolumn{3}{|c|}{ AAC group } & \multicolumn{3}{|c|}{ Non-AAC group } \\
\hline & Preoperative & postoperative & $P$ value & Preoperative & postoperative & $P$ value \\
\hline \multicolumn{7}{|l|}{ Visual acuity } \\
\hline UDVA (decimal value) & $0.25 \pm 0.23$ & $0.37 \pm 0.25$ & 0.0177 & $0.18 \pm 0.25$ & $0.38 \pm 0.27$ & 0.0012 \\
\hline CDVA (decimal value) & $0.37 \pm 0.34$ & $0.55 \pm 0.35$ & 0.0143 & $0.30 \pm 0.26$ & $0.49 \pm 0.30$ & 0.0260 \\
\hline $\mathrm{SE}$ (D) & $-0.97 \pm 1.18$ & $-0.15 \pm 0.78$ & 0.0017 & $0.59 \pm 0.75$ & $-0.07 \pm 0.75$ & 0.0447 \\
\hline IOP $(\mathrm{mmHg})$ & $39.46 \pm 16.71$ & $13.86 \pm 6.02$ & 0.0000 & $15.88 \pm 5.68$ & $14.82 \pm 5.70$ & 0.0890 \\
\hline
\end{tabular}
Data shown were presented as mean $\pm \mathrm{SD}$, Student-t test, $\mathrm{n}=17$ in each group. $P$ : preoperative vs postoperative. UDVA, uncorrected distance visual acuity; CDVA, corrected distance visual acuity; SE, spherical equivalent; IOP, intraocular pressure; D, diopters; AAC, acute angle closure. 
Table 3. Comparison of ocular parameters of the participants included in this study.

\begin{tabular}{|c|c|c|c|c|c|c|c|c|}
\hline \multirow[t]{2}{*}{ Parameters } & \multicolumn{3}{|c|}{ AAC group } & \multicolumn{3}{|c|}{ Non-AAC group } & \multirow[b]{2}{*}{${ }^{2} P$ value } & \multirow[b]{2}{*}{${ }^{3} P$ value } \\
\hline & $\begin{array}{c}\text { Affected } \\
\text { eyes }\end{array}$ & Fellow eyes & ${ }^{1} P$ value & $\begin{array}{l}\text { Affected } \\
\text { eyes }\end{array}$ & Fellow eyes & ${ }^{1} P$ value & & \\
\hline \multicolumn{9}{|l|}{$\begin{array}{l}\text { Ultrasound } \\
\text { Biomicroscopy }\end{array}$} \\
\hline ACD (mm) & $1.75 \pm 0.62$ & $2.39 \pm 0.65$ & 0.0060 & $3.24 \pm 0.98$ & $3.81 \pm 0.92$ & 0.0920 & 0.0000 & 0.0000 \\
\hline $\begin{array}{l}\text { AOD500 } \\
(\mathrm{mm})\end{array}$ & $0.07 \pm 0.35$ & $0.25 \pm 0.14$ & 0.0000 & $0.52 \pm 0.13$ & $0.54 \pm 0.11$ & 0.8275 & 0.0000 & 0.0000 \\
\hline LV (mm) & $1.59 \pm 0.12$ & $0.57 \pm 0.75$ & 0.0000 & $0.36 \pm 0.12$ & $0.41 \pm 0.10$ & 0.2364 & 0.0000 & 0.0555 \\
\hline I-curve (mm) & $0.14 \pm 0.05$ & $0.12 \pm 0.07$ & 0.3736 & $0.10 \pm 0.11$ & $0.11 \pm 0.15$ & 0.8014 & 0.1501 & 0.8014 \\
\hline IT750 (mm) & $0.36 \pm 0.03$ & $0.37 \pm 0.07$ & 0.7180 & $0.35 \pm 0.05$ & $0.34 \pm 0.05$ & 0.6923 & 0.2745 & 0.1150 \\
\hline $\begin{array}{l}\text { Zonular } \\
\text { compromise } \\
\text { (laxity or } \\
\text { loss) (No.) }\end{array}$ & 17 & 0 & 0.0000 & 17 & 0 & 0.0000 & 1.0000 & 0.1510 \\
\hline $\begin{array}{l}1 \text { quadrant } \\
\text { (No.) }\end{array}$ & 15 & 0 & 0.0000 & 12 & 0 & 0.0000 & 0.7073 & 0.1876 \\
\hline $\begin{array}{l}2 \\
\text { quadrants } \\
\text { (No.) }\end{array}$ & 2 & 0 & & 5 & 0 & & & \\
\hline $\begin{array}{l}\geq \\
\text { quadrants } \\
\text { (No.) }\end{array}$ & 0 & 0 & & 0 & 0 & & & \\
\hline \multicolumn{9}{|l|}{ Gonioscopy } \\
\hline $\begin{array}{l}\text { Modified } \\
\text { Shaffer } \\
\text { grade, 0-4 }\end{array}$ & $1.49 \pm 0.69$ & $3.07 \pm 0.63$ & 0.0000 & $3.70 \pm 0.47$ & $3.59 \pm 0.51$ & 0.4880 & 0.0000 & 0.0132 \\
\hline \multicolumn{9}{|l|}{ IOL Master } \\
\hline $\mathrm{AL}(\mathrm{mm})$ & $23.68 \pm 0.72$ & $23.91 \pm 1.15$ & 0.2771 & $25.75 \pm 2.57$ & $25.78 \pm 2.71$ & 0.7210 & 0.0001 & 0.0010 \\
\hline \multicolumn{9}{|l|}{$\begin{array}{l}\text { Other clinical } \\
\text { data }\end{array}$} \\
\hline $\begin{array}{l}\text { Lens nucleus } \\
\text { opacity, } \\
\text { LOCS III }\end{array}$ & $2.34 \pm 0.40$ & $2.12 \pm 0.28$ & 0.3285 & $2.15 \pm 0.68$ & $2.15 \pm 0.29$ & 0.4948 & 0.6475 & 0.7675 \\
\hline $\mathrm{C} / \mathrm{D}$ ratio & $0.31 \pm 0.04$ & $0.30 \pm 0.03$ & 0.8019 & $0.29 \pm 0.03$ & $0.29 \pm 0.04$ & 0.6832 & 0.3420 & 0.2172 \\
\hline $\begin{array}{l}\text { RNFL } \\
\text { thickness } \\
(\mu \mathrm{m})\end{array}$ & $97.75 \pm 15.33$ & $110.59 \pm 13.85$ & 0.1260 & $110.37 \pm 7.48$ & $107.18 \pm 11.91$ & 0.7240 & 0.0057 & 0.0242 \\
\hline
\end{tabular}

Data shown were presented as mean \pm SD, Mann-Whitney test and Student-t test, $n=17$ in each group. ${ }^{1} P$ : affected eyes vs fellow eyes. ${ }^{2} P$ : AAC group vs the non-AAC group in affected eyes; ${ }^{3} P$ : AAC group vs the non-AAC group in fellow eyes. Respectively, anterior segment parameters and zonular evaluation were measured by ultrasound biomicroscopy. Axial length was measured by IOL Master. Grading of anterior chamber angle was evaluated by gonioscopy. Lens nucleus opacity and C/D ratio were observed in slit lamp. RNFL thickness was measured by optical coherence tomography. ACD, anterior chamber depth; AOD500, angle opening distance at $500 \mu \mathrm{m}$ from scleral spur; LV, lens vault; I-curve, iris curvature; IT750, iris thickness at $750 \mu \mathrm{m}$ from scleral spur; AL, axial length; LOCS III, Lens Opacities Classification System III; C/D ratio, Cup/Disk ratio; RNFL, retinal nerve fiber layer; AAC, acute angle closure.

\section{Figures}




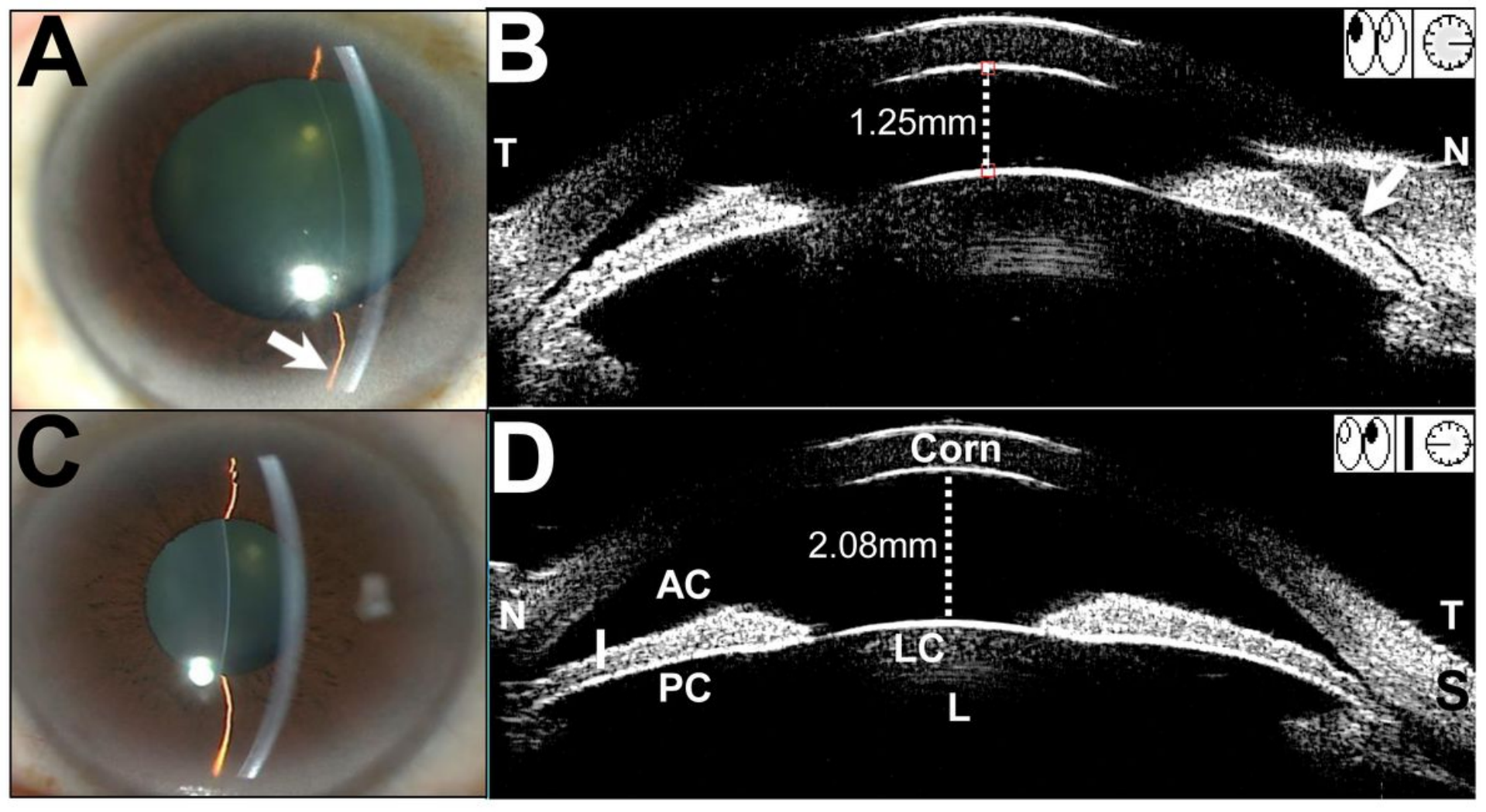

\section{Figure 1}

Clinical characteristics of a lens subluxation patient with AAC attack. (A) Affected eye (right eye): Slit-lamp biomicroscopy demonstrated that the anterior chamber depth (ACD) was shallow in the central region, and it was shallower in the periphery (arrow). Imaging of Ultrasound Biomicroscopy (UBM) showed $1.25 \mathrm{~mm}$ central ACD, while the peripheral nasal ACD was shallower (arrows). The lens vault (LV) was $1.48 \mathrm{~mm}$. (B) Fellow eye (left eye): The central and peripheral ACD was wider than the affected eye. The central ACD was $2.08 \mathrm{~mm}$ and the lens vault (LV) was $0.95 \mathrm{~mm}$. The LV is defined as the perpendicular distance between the anterior pole of the crystalline lens and the horizontal line joining the two scleral spurs. AAC, acute angle closure; Corn, cornea; S, sclera; I, iris; PC, posterior chamber. 